University of Wollongong

Research Online

Australian Institute for Innovative Materials -

Papers

Australian Institute for Innovative Materials

$1-1-2015$

Mechanism of periodic height variations along self-aligned VLS-grown planar nanostructures

\author{
Julian Steele \\ University of Wollongong, js598@uowmail.edu.au \\ Josip Horvat \\ University of Wollongong, jhorvat@uow.edu.au \\ Roger A. Lewis \\ University of Wollongong, roger@uow.edu.au \\ M Henini \\ University of Nottingham \\ D. Fan \\ University of Arkansas
}

See next page for additional authors

Follow this and additional works at: https://ro.uow.edu.au/aiimpapers

Part of the Engineering Commons, and the Physical Sciences and Mathematics Commons

Research Online is the open access institutional repository for the University of Wollongong. For further information contact the UOW Library: research-pubs@uow.edu.au 


\title{
Mechanism of periodic height variations along self-aligned VLS-grown planar nanostructures
}

\begin{abstract}
In this study we report in-plane nanotracks produced by molecular-beam-epitaxy (MBE) exhibiting lateral self-assembly and unusual periodic and out-of-phase height variations across their growth axes. The nanotracks are synthesized using bismuth segregation on the $\mathrm{GaAsBi}$ epitaxial surface, which results in metallic liquid droplets capable of catalyzing GaAsBi nanotrack growth via the vapor-liquid-solid (VLS) mechanism. A detailed examination of the nanotrack morphologies is carried out employing a combination of scanning electron and atomic force microscopy and, based on the findings, a geometric model of nanotrack growth during MBE is developed. Our results indicate diffusion and shadowing effects play significant roles in defining the interesting nanotrack shape. The unique periodicity of our lateral nanotracks originates from a rotating nucleation "hot spot" at the edge of the liquid-solid interface, a feature caused by the relative periodic circling of the non-normal ion beam flux incident on the sample surface, inside the MBE chamber. We point out that such a concept is divergent from current models of crawling mode growth kinetics and conclude that these effects may be utilized in the design and assembly of planar nanostructures with controlled non-monotonous structure
\end{abstract}

\section{Keywords}

grown, planar, along, variations, height, vls, periodic, mechanism, aligned, nanostructures, self

\section{Disciplines}

Engineering | Physical Sciences and Mathematics

\section{Publication Details}

Steele, J. A., Horvat, J., Lewis, R. A., Henini, M., Fan, D., Mazur, Y. I., Dorogan, V. G., Grant, P. C., Yu, S., Salamo, G. J. et al (2015). Mechanism of periodic height variations along self-aligned VLS-grown planar nanostructures. Nanoscale, 7 (48), 20442-20450.

\section{Authors}

Julian Steele, Josip Horvat, Roger A. Lewis, M Henini, D. Fan, Yu. I. Mazur, V G. Dorogan, P C. Grant, S.-Q $\mathrm{Yu}$, and G J. Salamo 


\title{
Nanoscale
}

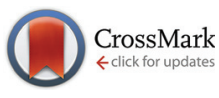

Cite this: Nanoscale, 2015, 7, 20442

\section{Mechanism of periodic height variations along self-aligned VLS-grown planar nanostructures}

\author{
J. A. Steele, ${ }^{\star a}$ J. Horvat, ${ }^{a}$ R. A. Lewis, ${ }^{a}$ M. Henini, ${ }^{b}$ D. Fan, ${ }^{c, d}$ Yu. I. Mazur, ${ }^{d}$ \\ V. G. Dorogan, ${ }^{d}$ P. C. Grant, ${ }^{\text {C }}$ S.-Q. Yu ${ }^{c, d}$ and G. J. Salamo ${ }^{d}$
}

In this study we report in-plane nanotracks produced by molecular-beam-epitaxy (MBE) exhibiting lateral self-assembly and unusual periodic and out-of-phase height variations across their growth axes. The nanotracks are synthesized using bismuth segregation on the GaAsBi epitaxial surface, which results in metallic liquid droplets capable of catalyzing GaAsBi nanotrack growth via the vapor-liquid-solid (VLS) mechanism. A detailed examination of the nanotrack morphologies is carried out employing a combination of scanning electron and atomic force microscopy and, based on the findings, a geometric model of nanotrack growth during MBE is developed. Our results indicate diffusion and shadowing effects play significant roles in defining the interesting nanotrack shape. The unique periodicity of our lateral nanotracks originates from a rotating nucleation "hot spot" at the edge of the liquid-solid interface, a feature caused by the relative periodic circling of the non-normal ion beam flux incident on the sample surface, inside the MBE chamber. We point out that such a concept is divergent from current models of crawling mode growth kinetics and conclude that these effects may be utilized in the design and assembly of planar nanostructures with controlled non-monotonous structure.

Received 25th September 2015 Accepted 6th November 2015

DOI: $10.1039 / c 5 n r 06676 j$

www.rsc.org/nanoscale challenges for growers; $\mathrm{Bi}$ exhibits a strong tendency to surface-segregate and form surface droplets. ${ }^{10}$ In the liquid phase, Bi droplets are known to ultimately act as catalysts to GaAs-based VLS growth. ${ }^{11}$

Numerous models have highlighted the importance that growth chemistry and parameters have on the VLS process, ${ }^{12}$ with several growth modes already identified (phase transitions, ${ }^{13,14}$ diameter oscillating, ${ }^{15,16}$ kinking, ${ }^{17,18}$ and crawling $^{14,19}$ ) as emerging from different interactions between the catalyst and underlying crystal. Nanostructures possessing non-monotonous morphological variations along the growth direction are offering new avenues for device engineering, with periodic dimensional changes ${ }^{13,16,20}$ of particular interest due to novel quantum confinement effects. ${ }^{21-24}$ Moreover, the formation of preferentially orientated structures ${ }^{25}$ are developed in parallel with advancing technological aims to employ bottom-up fabrication techniques. ${ }^{19,26-29}$ Nanowires which self-align on the surface could play an important role in combining the synthesis and assembly in a single step, while offering compatibility with traditional planar device structures. ${ }^{25,28-31}$

In this paper, we extend the current palette of non-monotonous one-dimensional nanostructures and investigate a new type of self-aligned GaAsBi planar nanotrack exhibiting periodic changes in height along its full length. We find that an explanation for this unusual shape is outside the capacity of existing planar nanowire growth $\operatorname{models}^{19,25}$ and develop a 
more pertinent semi-empirical growth model - one that accounts for the localized adsorption of species on the droplet surface and connects them to a functional describing the rate of nucleation across an inhomogeneous liquid-solid growth interface. Our results indicate that the unusual morphology arises as a consequence of large diffusion and shadowing effects, influences that are typically avoided during nanowire growth. We conclude that these effects may actually be utilized in the controlled preparation of planar nanowires with interesting periodic structure.

\section{Experimental procedure}

We will define our in-plane nanostructures as nanotracks (rather than nanowires, given their unique aspect ratio; see Fig. 1 and 2). Performed in a standard Riber 32P MBE system on a GaAs (001) wafer, the sample was first heated to $610{ }^{\circ} \mathrm{C}$ for 10 minutes to desorb the native oxide layer, then a $400 \mathrm{~nm}$ GaAs buffer layer was grown at $580{ }^{\circ} \mathrm{C}$ and a growth rate of 1 monolayer per second. Following a 20 min growth interruption during which the sample was cooled to $325-330^{\circ} \mathrm{C}$ and the growth rate lowered to $\sim 0.1$ monolayer per second, the nanotracks formed during the growth of an uncapped, $300 \mathrm{~nm}$ thick (001) GaAsBi film. A relatively large Bi flux was used, twice that of As, while maintaining an approximately equal flux of As and Ga. This places the growth conditions well within the Bi saturation regime, ${ }^{10}$ where an alloying limit is imposed by the low miscibility of Bi into GaAs. As a consequence, liquid Bi droplets form (Fig. 1(a)) through aggregation of unincorporated $\mathrm{Bi}$ atoms on the surface. The growth temperature is relatively low as compared with the MBE growth of other III-V compounds, however it is still well above the Ga-Bi eutectic point $\left(222^{\circ} \mathrm{C}\right.$ ) and the low melting point (as compared to other group-V metals) of pure $\mathrm{Bi}$ metal $\left(271{ }^{\circ} \mathrm{C}\right)$.
While the local Bi droplet density varied slightly across the sample surface, the size and typical surface effects did not. Our MBE geometry consists of standard non-normal MBE effusion cells along with sample rotation to promote homogeneous epitaxial incorporation of $\mathrm{Bi}$, both of which will be quantified later. The nominal bismuth concentration in the $\mathrm{GaAs}_{1-x} \mathrm{Bi}_{x}$ epilayer is $x=0.040$, determined through evaluating the photoluminescence (PL) peak energy. ${ }^{32}$ When compared to other droplet free locations on the sample surface, this value is found to be consistent and comparable to known Bi saturation limits for $\mathrm{GaAs}_{1-x} \mathrm{Bi}_{x}$ alloys grown under similar conditions. ${ }^{33}$

Electron dispersion spectroscopy (EDS) measurements indicate that $\mathrm{Bi}$ is successfully incorporated into the nanotracks. The extent of Bi alloying in the nanotracks is $70-80 \%$ of that incorporated into the epitaxial layer: $x_{\text {nanotrack }}=0.028-0.032$. This interesting compositional aspect of nanotrack growth is not considered further here and is the focus of a separate investigation.

\section{Results and discussion}

\subsection{Morphological characterization of in-plane GaAsBi nanotracks}

Fig. 1(a) shows a representative scanning electron micrograph (SEM) of the GaAsBi sample surface, revealing a relatively smooth GaAsBi epitaxial growth overgrown by metallic surface droplets and the formation of in-plane nanotracks. EDS measurements indicated pure Bi droplets are formed on the GaAsBi surface (no final Ga/Bi dual composites ${ }^{10}$ ), which is indicative of Bi-rich growth and attributed to Bi segregation. ${ }^{10}$ The bottom inset in Fig. 1(a) focuses on the parallel/antiparallel alignment of the nanotracks and the contrasting forms by which they can terminate; with or without the surface droplet catalyst. The pressure of $\mathrm{Bi}$ is high at $330^{\circ} \mathrm{C}$ and a sufficiently
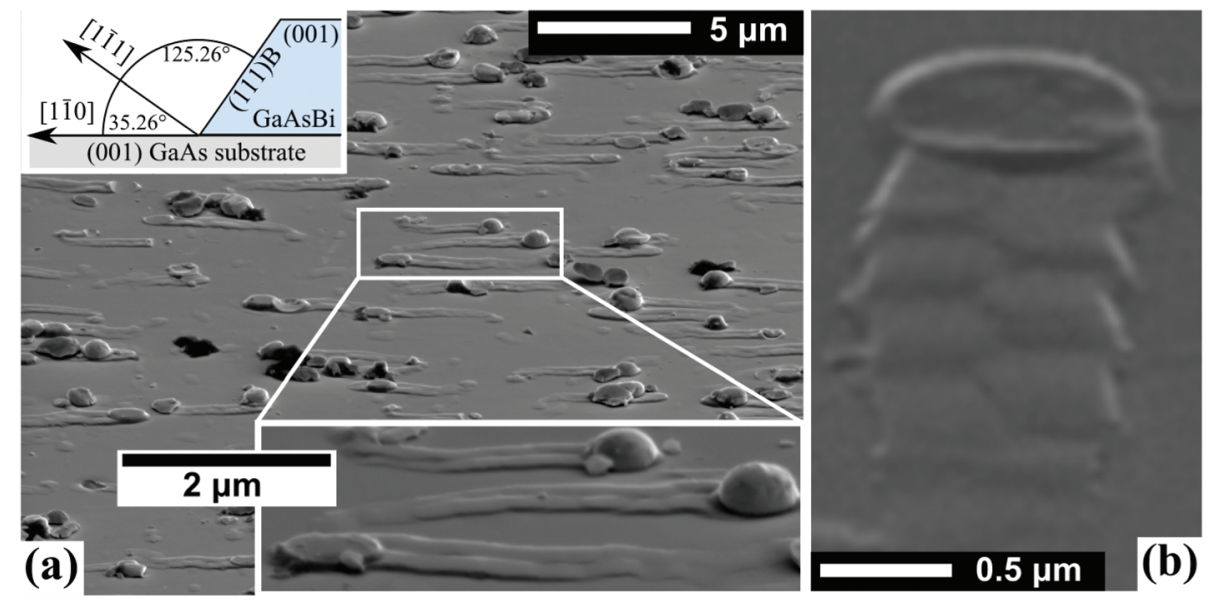

Fig. 1 Secondary electron SEM images of (a) GaAsBi sample surface, showing the formation of surface droplets and trailing nanotracks. The bottom inset here presents an enlargement of the SEM image and exhibits the parallel/antiparallel nature of nanotrack growth, while top inset provides geometric information of relevant crystal faces relative to the [001] growth direction. (b) An alternative perspective of nanotrack formation, revealing pronounced out-of-phase height variations across the growth axes. 

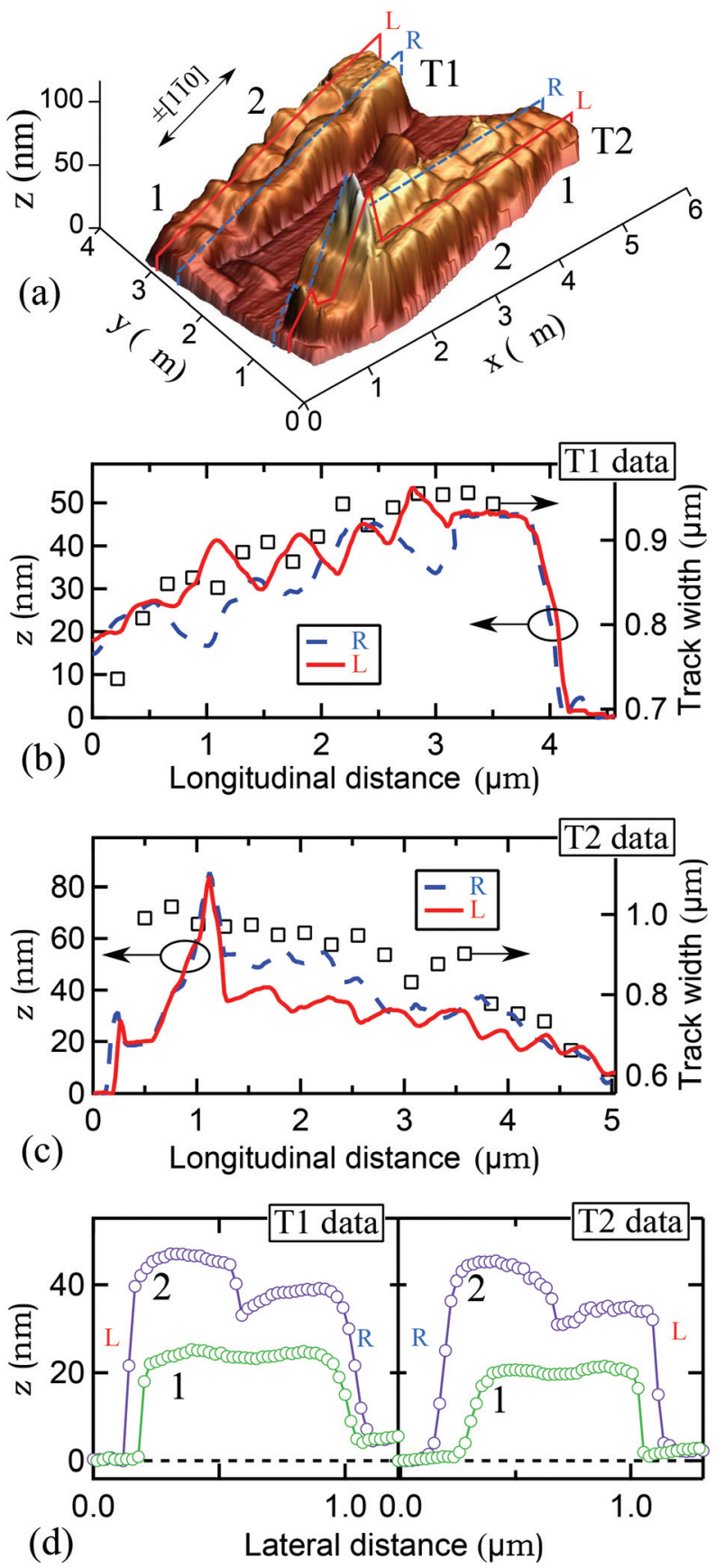

Fig. 2 (a) AFM micrograph of two antiparallel nanotracks, labeled T1 and T2. The two axial lines along both nanotracks (one solid red and the other broken blue) indicate the paths of the line height scans presented in (b) and (c). The nanotrack width data in (b) and (c) were determined via multiple line height scans measured orthogonally to $\pm[1 \overline{1} 0]$, and (d) shows two of these scans obtained for both nanotracks at the locations indicated in (a).

large $\mathrm{Bi}$ beam equivalent pressure (BEP) is required to avoid total evaporation of Bi droplets. If the Bi BEP drops well below the Bi desorption rate, none of the bulk Bi will remain. That many nanotracks terminate forming nanodiscs ${ }^{11}$ without droplets reflects a partial disruption of this fine balance, likely towards the final stages of growth.

Of one hundred nanotracks inspected, the typical lengths (4-5 $\mu \mathrm{m})$, heights ( $45-55 \mathrm{~nm})$, as well as the terminating nanodisc diameters $(\sim 940 \mathrm{~nm})$, appear steady across the whole sample surface. Such a consistent mode of distribution indicates a uniform growth environment, as well as signifying our Bi liquid droplets necessitate a critical volume before moving. In the absence of direct observation, determining the initial time of nanotrack formation is difficult. We note that nanotracks formed early on during growth would likely exhibit an overgrown character, due to the rising (and much thicker) epitaxial layer. That all nanotracks possess comparable dimensions, and none appear to be overgrown, suggests a temporally unified deposition of the nanotracks. Further, simultaneous growth likely occurred late during MBE.

Fig. 1(b) offers a different perspective of the nanotracks. Topologically-sensitive secondary electron imaging (SEI) is employed to highlight the presence of a periodic undulation pattern seen across the nanotrack center, evidenced by image shadowing. The variation in height exhibited here is archetypal of all inspected nanotracks, a characteristic persisting along the entire nanotrack length. Another fascinating feature is the center axial line of the nanotrack. As far as we can tell, planar nanostructures (nanowires, or otherwise) demonstrating this unusual morphology are yet to be reported.

Unlike for free-standing nanowires, a unified understanding of crawling modes is not yet realized. ${ }^{19,25,26}$ Principally, the VLS mechanism ${ }^{2}$ underpins our observations of Bi droplet movement and the formation of self-aligning structures. Such VLS driven growth should not be surprising during the Bi-rich MBE of GaAsBi alloys; Schwarz et al. ${ }^{19}$ have calculated the stability of different modes of steady-state growth and found a uniquely broad stability range for the promotion of crawling modes, in which lateral, rather than free-standing, nanowires form. While the kinetics of VLS growth are extremely complicated, our aim is to introduce the fundamental processes in aid of interpreting our results.

With the Bi droplet in the liquid phase, the eutectic will adsorb $\mathrm{Ga}$ and As species from a vapor with a higher chemical potential $\left(\mu_{\mathrm{v}}\right)$, relative to the liquid chemical potential $\left(\mu_{1}\right)$. Thus the difference in these quantities is defined as

$$
\Delta \mu_{\mathrm{vl}}=\mu_{\mathrm{v}}-\mu_{\mathrm{l}},
$$

where the system is said to be supersaturated (concentration of the components in the liquid phase is higher than the equilibrium concentration) for $\Delta \mu_{\mathrm{vl}}>0$. In a state of supersaturation the droplet may equilibrate via an exchange at the liquidsolid interface, rather than desorbing already deposited material back into the vapor, if the chemical potential of the solid $\left(\mu_{\mathrm{s}}\right)$ is lower than $\mu_{\mathrm{l}}$. In response to the concentration imbalance the droplet crystallizes material at the liquid-solid interface; an illustration of this process is provided in Fig. 3. With a continual supply of vapor, and growth of the epilayer in 


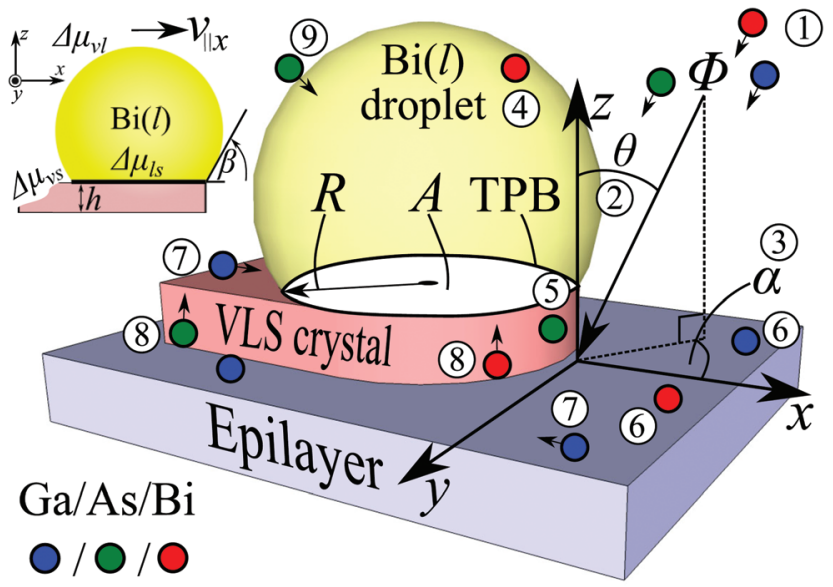

Fig. 3 (Color online) Schematic illustrations of geometric model with the parameters described in the text (the processes described in the image are not atomic species specific): (1) incident ion beam flux $(\Phi)$ and mass transport in the gas phase; (2) the direction of the incoming nonnormal flux is angled $\theta$ away from the growth axis ( $z$-axis); (3) the sample is constantly rotated during MBE, forming angle $\alpha$ with the nanotrack growth direction; vapor impinging on the (4) droplet, (5) nanotrack sidewall, and (6) the epilayer surface; diffusion of atoms across the (7) epilayer surface and (8) nanotrack sidewall; (9) chemical reaction at the vapor-liquid interface and adsorption of vapor species. The inset shows the relative velocity $\left(v_{\| x}\right)$ of the droplet, the nanotrack height $h$, and the wetting angle $\beta$, which meets at the three phase boundary (TPB).

the solid-state proceeding relatively slowly, herein lies the driving force of the VLS mechanism: $\mu_{\mathrm{v}} \geq \mu_{1} \geq \mu_{\mathrm{s}} \cdot{ }^{34}$

Generally, nucleation at the liquid-solid interface will be preferentially faceted and growth - as well as lateral motion will proceed perpendicular to the dominant crystal face. The tendency for facets with low surface energies to form more readily gives rise to self-alignment, evidenced by only a small fraction of our nanotracks taking on nonconforming paths. The mobility of the liquid surface at the liquid-solid interface means it is accommodating to non-(001) facet planes which do not grow along the $c$-axis. Subsequently, the growth direction will often correspond to the lowest energy facet of the crystal being synthesized. With simultaneous growth of multiple facets of equivalent energy the final growth direction is ultimately determined by random small-scale topological or thermal fluctuations. A crawling growth mode then propagates with the liquid droplet pinned to its front via surface tension.

To investigate our preferred growth directions, polarized micro-Raman backscattering data were recorded from the (001) sample surface and the intensity of the optical modes was analyzed as a function of scattering geometry. The measurements indicated that the nanotracks align with either the $\pm[110]$ or $\pm[1 \overline{1} 0]$ crystallographic directions. From a crystallographic point of view, the orthogonal $\pm[110]$ and $\pm[1 \overline{1} 0]$ directions on a (001)-oriented zincblende substrate are not equivalent to each other, and growth will occur where interfacial energy is minimized. In zincblende GaAsBi, group-V terminates on (111)B facets, which has the lowest free energy. ${ }^{35}$
The top inset of Fig. 1(a) shows the out-of-plane [111]B direction (also written as [111]). Given this directional growth property, and the lateral projection of [111]B onto the epitaxial surface, we assign $\pm[1 \overline{1} 0]$ as the preferential growth direction for our nanotracks. This bi-directional growth facet agrees well with the literature. ${ }^{25,30,31,36}$ That we see equal movement in opposite directions eliminates thermal gradient or electric field effects underpinning this preference.

The typical details of the nanotrack morphology are contained in the AFM data shown in Fig. 2(a), displaying two antiparallel planar nanotracks, side-by-side. At this aspect ratio the central line and height variations along the nanotracks become vivid. The terminating form of $\mathrm{T} 2$ is very different to the flat nanodisc of $\mathrm{T} 1$ and similar to that observed in the synthesis of in-plane nanowires, ${ }^{26,28}$ and asymmetric nanoholes, ${ }^{37}$ counting its preferential assembly of a "pit" along the $\pm[110]$ direction. Nanotracks terminating in this way - with a high raised lips on the trailing side - are uncommon and only noted here for completeness.

An analysis of the AFM image (using the WSxM v5.0 software ${ }^{38}$ ) is presented in Fig. 2(b)-(d) for both nanotracks, with the paths taken for the line height scans indicated in Fig. 2(a). These data are typical of all nanotracks surveyed, with several pervasive morphological features.

The width of the nanotracks steadily increases perpendicular to the growth direction by roughly $20 \%$ over its whole length, data for which is presented in Fig. 2(b) and (c). This is paralleled by a gradual increase in height. Within the framework of VLS processes, one can expect that the size and position of the Bi droplet is related to the width and position of the nanotrack; the liquid-solid interface is confined to the area of precipitated crystal. The increase in our nanotrack width is analogous to tapered nanowire which are shaped by a changing catalyst size during growth. ${ }^{39}$ Previous studies have reported an increase in droplet size during motion and this particular aspect of growth kinetics appears to define the resulting width - and, in turn, height ${ }^{40}$ - of our nanotracks.

A periodic variation in the nanotrack height appears the full length of growth, peaking both at the far left (L) and right (R) hand sides. The line height data in Fig. 2(b) and (c) best communicates this nanotrack characteristic, with T1 and T2 together exhibiting comparable periods and amplitudes. In fact, once the steady gain in height is accounted for, the rising and falling angles are found to be even, and approximately equal to $1.5^{\circ}$. Note that the undulation height and period exhibited on the L side (in both $\mathrm{T} 1$ and $\mathrm{T} 2$ ) appears more regular and cyclic. In contrast, the $\mathrm{R}$ side exhibits more disorder in these features. The sidewall gradients of the $\mathrm{L}$ and $\mathrm{R}$ sides also significantly differ, with a steeper lateral rise consistently seen for the L side (see Fig. 2(d)). Regarding the relative lateral position of peaks and troughs, they appear approximately out-of-phase across the nanotrack center. However, this particular morphological feature is made less distinct here due to the disorder of the $\mathrm{R}$ side. For reference, we point out that this growth aspect is evidently contained in the SEM data shown in Fig. 1(b). 
The high aspect ratio in which Fig. $2(\mathrm{~d})$ is presented $(z: x$, $y \approx 1: 37$ ) reveals a pronounced centered dip in the cross section of the nanotrack, forming an "M-shape". The central depression in height is less defined at the beginning of the nanotrack growth (traces labeled 1), sharpening up with an increase in nanotrack height and width (traces labeled 2).

\subsection{Geometric modeling of nanotrack morphology}

We present now a simple geometric growth model to lend insight to our experimental observations. The numerics of the model were carried out using Matlab R2015a software.

Geometric models describing nanowire growth rates often monitor only the total rate of species arriving at the droplet surface and assume that growth at the liquid-solid interface proceeds uniformly. For a liquid droplet creating a homogeneous growth interface with the underlying crystal, it follows that lateral motion would only produce a simple and relatively featureless planar structure. To explain our interesting nanotrack morphology it is clear that a meaningful model must account for the localization of adsorbed species and an inhomogeneous growth interface. The main parameters and limiting processes of our model are summarized in Fig. 3 and are discussed in detail below.

We consider a rigid circular liquid-solid interface of radius $R$ and, by studying key supply restrictions, form a dynamic functional describing the nucleation rate over its area, $A$. Central to the model are the two different modes of species supply: (i) direct impingement from a rotating non-normal beam-like vapor source (creating angle $\theta$ with the $z$-axis), which is shadowed by the $3 \mathrm{D}$ droplet surface, and (ii) diffusion from various paths crossing the solid-state to reach the moving droplet. For simplicity, we only consider mode (i) to be dynamic since it must account for a constantly changing azimuthal angle, $\alpha$. It follows that a steady-state solution may be estimated to account for diffusion, mode (ii), relative to the lateral droplet velocity, $v_{\| x}$ (see Fig. 3 ).

Our model reduces the supply problem to a single type of limiting growth species, the arrival of which at the liquid droplet surface will help to define the nucleation rate at the liquid-solid interface. For the synthesis of III-V semiconductor nanowires (planar or vertical) this is not an oversimplification, given that group-III Ga species presumably limit growth kinetics. $^{41}$ In this way, only a single set of $\theta$ and $\alpha$ values are required.

3.2.1 Theoretical consideration. The vapor flux can impinge onto the substrate, the nanotrack sidewall and surface, as well as directly onto the liquid droplet surface. The coordinate-dependent concentrations of stationary adatoms on the crystal substrate $\left(n_{\mathrm{s}}\right)$ and the nanotrack sidewall $\left(n_{\mathrm{t}}\right)$ surfaces obey the diffusion equations

$$
\begin{aligned}
& D_{\mathrm{s}} \Delta n_{\mathrm{s}}+\Phi \cos (\theta)=\frac{n_{\mathrm{s}}}{\tau_{\mathrm{s}}}, \\
& D_{\mathrm{t}} \frac{\mathrm{d}^{2} n_{\mathrm{t}}}{\mathrm{d} z^{2}}+\Phi \sin (\theta)=\frac{n_{\mathrm{t}}}{\tau_{\mathrm{t}}}
\end{aligned}
$$

Here $\Delta$ is the $2 \mathrm{D}$ in-plane Laplace operator, $D_{\mathrm{s}}, D_{\mathrm{t}}$ are the diffusion coefficients (with units of $\mathrm{m}^{2} \mathrm{~s}^{-1}$ ) for adatoms on the substrate and sidewalls, respectively. The second terms in these equations define the arrival rate of adatoms from the impinging ion beam flux $\Phi$ (incident at angle $\theta$ to the surface normal), while $\tau_{\mathrm{s}}$ and $\tau_{\mathrm{t}}$ are the effective lifetimes of diffusing adatoms on the substrate and nanotrack sidewalls, respectively. The numerical values of $\tau_{\mathrm{s}}$ and $\tau_{\mathrm{t}}$ (with units of $\mathrm{s}$ ) are principally governed by the rates of surface nucleation. We assume that the surface diffusion process (scheme 7 and 8 in Fig. 3; first terms in eqn (2) and (3)) possesses radial symmetry. Symmetry then dictates that the growth chronology will critically depend on the magnitudes of the adatom diffusion lengths on the epilayer surface

$$
\lambda_{\mathrm{s}}=\sqrt{D_{\mathrm{s}} \tau_{\mathrm{s}}}
$$

and on the nanotrack sidewall surface

$$
\lambda_{\mathrm{t}}=\sqrt{D_{\mathrm{t}} \tau_{\mathrm{t}}}
$$

Important to our model are simply the lifetimes and diffusion lengths of adatoms on the crystal surfaces, which are set relative to the droplet speed, $v$, using a scaling factor. There will be large difference between these two diffusion lengths; the solid-state reaction on the epilayer (MBE) will create a surface which is more atomically flat and less defective than the highly faceted nanotrack surface. After considering rates of impingement, we proceed to flatten the diffusion path of adatoms into two dimensions and simply consider a high diffusion barrier $\left(\lambda_{\mathrm{t}} \ll \lambda_{\mathrm{s}}\right)$ imposed by the rough nanotrack crystal surface, of length $h$, in the $x-y$ plane. This diffusion barrier also exists in the trailing droplet path.

For the impinging flux of the droplet surface, a simplified hemispherical geometry ${ }^{42}$ (droplet wetting angle of $\beta=\pi / 2$ ) is adopted where the droplet interception of flux is

$$
I_{\mathrm{Bi}(1)}=\frac{1+\cos (\theta)}{2} \pi R^{2} \Phi .
$$

We ignore the diffusion of species over the "rough"2 liquid surface and assume the existence of a sufficiently high number of accommodation sites over its entire area. Using eqn (6) and the projection of $I_{\mathrm{Bi}(1)}$ onto the $x-y$ plane (via radial diffusion within the liquid droplet), we then arrive at a steadystate estimate for a fixed $\alpha$.

Employing a general form ${ }^{43}$ to define the dependence of the nucleus formation rate on the various system factors, we have

$$
J=\omega Z C \exp \left(-\Delta G / k_{\mathrm{B}} T\right),
$$

where $\omega$ is the frequency of attachment of growth species at the interface and is dependent on the number of nucleation sites, $Z$ is a statistical dimensionless number known as the Zeldovich factor, ${ }^{44}$ which is dependent on the change in the Gibbs free energy of nucleation, $\Delta G$. In addition $k_{\mathrm{B}} T$ is the thermal energy and $C$ indicates the magnitude of the concentration of the relevant species in the liquid (not to be confused 
with $n_{\mathrm{s}}$ or $n_{\mathrm{t}}$ ), and is related to the supersaturation and the equilibrium concentration $\left(C_{\text {eq }}\right)$ by

$$
C=C_{\text {eq }} \exp \left(\Delta \mu_{1 \mathrm{~s}} / k_{\mathrm{B}} T\right) .
$$

When $C_{\text {eq }}$ is zero, the supersaturation and nucleation rate at the liquid-solid interface, are zero. Thus it follows that for a constant temperature, an increase in the concentration $C$ produces a direct rise in the nucleation rate $J$ through eqn (7) (assuming an unchanging critical nucleus size).

We must now consider the important difference between nucleation at the center of the solid-liquid interface and at the bordering three phase boundary (TPB). ${ }^{45} \mathrm{~A}$ minimization of the nucleation barrier (Gibbs free energy) at TPB means it is the preferred nucleation site in a three phase system; ${ }^{34}$ Sear $^{46}$ demonstrated that nucleation rate at a TPB can be orders of magnitude higher than growth at the other surfaces and interfaces. With adatoms essentially only diffusing across the solid crystal surface, supersaturation is also at its highest at the TPB because it is in direct contact with species supply. Growth near the TPB edge also facilitates non-faceted growth, further roughening the crystal surface. In this case, the supersaturation required for growth near the TPB may actually be further reduced. The VLS synthesis of relatively narrow nanowires (diameters in the order of tens of $\mathrm{nm}$ ) is considered piecewise, ${ }^{47}$ as a nucleus at the TPB (or liquid-solid interface) spreads out in steps. These factors contribute to enhanced growth near TPB edge, which is necessary to explaining our observations. Within the context of our model, this implies that the nucleation rate at the bordering TPB will not only be at its highest, but also locally enhanced and dispersed towards the interface center. Thus, we employ a ring-shaped one-sided Gaussian function to describe the elevated growth rate in this region. This function peaks at the TPB (distance $R$ ) with the parameters adjusted to obtain the proportions of the growth structures that correspond to the observed proportions.

3.2.2 Empirical inputs, model description, and simulated data. In reliably estimating the relative nucleation rate across $A$, for a given $\alpha$, we depend on empirical data of the nanotrack morphology. Firstly, the sample was continually rotated in the MBE growth chamber at a rate of 5 revolutions per minute. Without direct in situ observations, ${ }^{48}$ it is expected the periodic appearance of peaks and troughs will have formed at equivalent values of $\alpha$ during repeated rotations (i.e. $\alpha=j 2 \pi$, where $j=1,2,3, \ldots)$. Thus analysis of the AFM data suggests the droplets move and deposit VLS crystal with a lateral speed of between 2.5 to $3 \mu \mathrm{m}$ per minute. This relatively high lateral growth rate is not unreasonable, given the relatively large area of $A,{ }^{40}$ nanotracks only reach typical heights of approximately $50 \mathrm{~nm}$, and that droplet movement here is in no way comparable to the vertical growth of nanowires; a droplet atop a growing vertical nanowire is moved entirely by displacing itself through VLS growth. The impact of high droplet mobility on the collection of surface diffusing adatoms is large; $v_{\| x}$ will induce, and scale with, a supply differential between the front and rear of the droplet. The number of diffusing atoms arriv- ing at the front of the droplet is enhanced by its relative motion. On the other hand, diffusing species reaching the rear of the droplet must traverse $h$ unaided and pass an additional distance across the atomically rough nanotrack top.

Our AFM experiments indicate the nanotracks experience an overall $\sim 20 \%$ steady increases in width during lateral growth. The size of $R$ defines the nanotrack width, thus increases linearly with lateral distance (centered on $y=0$; see Fig. 3) within our model. In fact, the measured lateral period the distance recorded between consecutive undulation peaks or troughs - is found to be close to the nanotrack width, and permits a relationship for these values in the model; the undulation period (defined by equivalent values of $\alpha$ ) is set equal to twice the radius.

Finally, the model considers a droplet that is sufficiently large enough to initiate and sustain VLS growth - the effects of the initiation of droplet movement on the nanotrack growth are not considered in the model. Hence the liquid droplet is already moving unilaterally with a constant speed and continually solidifies crystal at the liquid-solid interface. Accepting these kinetic features, we estimate a $2 \mathrm{D}(x-y$ plane $)$ steady-state dynamic nucleation rate across $A$ for growth in the $z$-direction, and for a given $\alpha$. Fig. 4 presents our normalized nucleation rate over $A$ for four fixed azimuthal angles covering $0 \leq \alpha \leq \pi$. From these plots it becomes obvious that the dynamic surface $A$ experiences a rotating nucleation "hot spot" and a persisting center line of low nucleation, existing at the center and extending out towards its rear. The latter is enhanced by the relatively strong nucleation rate near the TPB, as well as the scarcity of diffusing supply species arriving at the very back of the moving droplet.
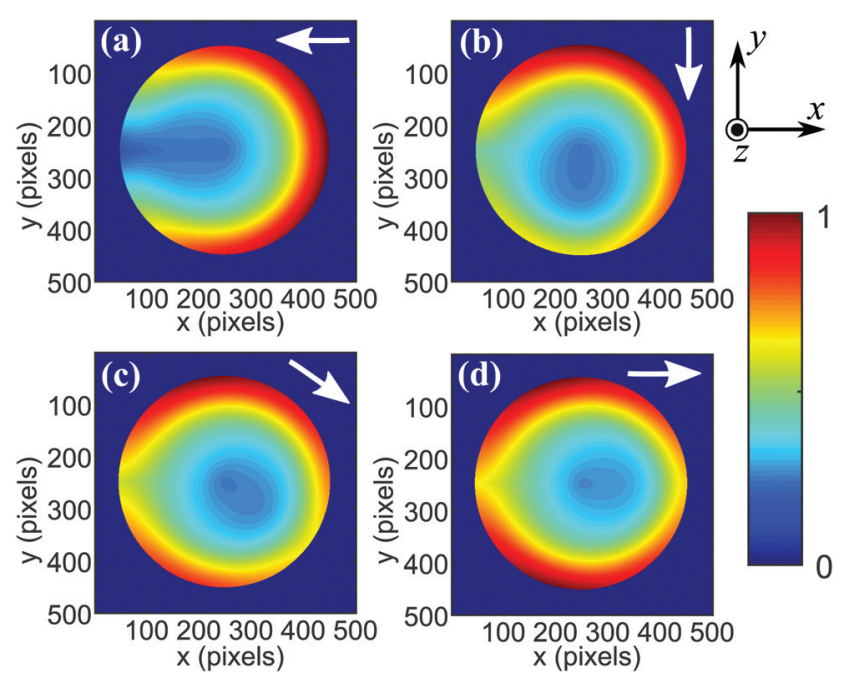

Fig. 4 (Color online) Normalized nucleation (in the $z$-direction) rate determined by the model at the liquid-solid interface $A$ for differing $\alpha$ values of (a) $0^{\circ}$, (b) $90^{\circ}$, (c) $145^{\circ}$, and (d) $180^{\circ}$. The depicted coordinate system relates to the axes shown in Fig. 3 and the white arrows indicate the relative flux directions for each angle. 
In simulating the final nanotrack morphology we essentially take a discrete approach: scaled matrix values of surface $A$ are cumulatively summed through iterations of a shifting center and rotating $\alpha$. A total of 2000 iterations are performed in this manner covering a distance just over $10 R$. The result of this procedure is presented in Fig. 5(a), with line scan analyses of the simulated nanotrack - analogous to that performed in Fig. 2 - displayed in Fig. 5(b) and (c). There is a good agreement between the simulation and experiment, with the typical nanotrack characteristics clearly present. The periodic and out-of-phase height variations along the nanotrack appear equally spaced across both $\mathrm{L}$ and $\mathrm{R}$ sides, with a height that steadily rises throughout growth.

Through our narrow definition of the droplet kinetics (including an increasing $R$ ) and of a lateral period (with its connection to the frequency of $\alpha$ ), it is not unexpected that we arrive at a result which strongly resembles experiment. What is interesting are the correct morphological subtleties existing in
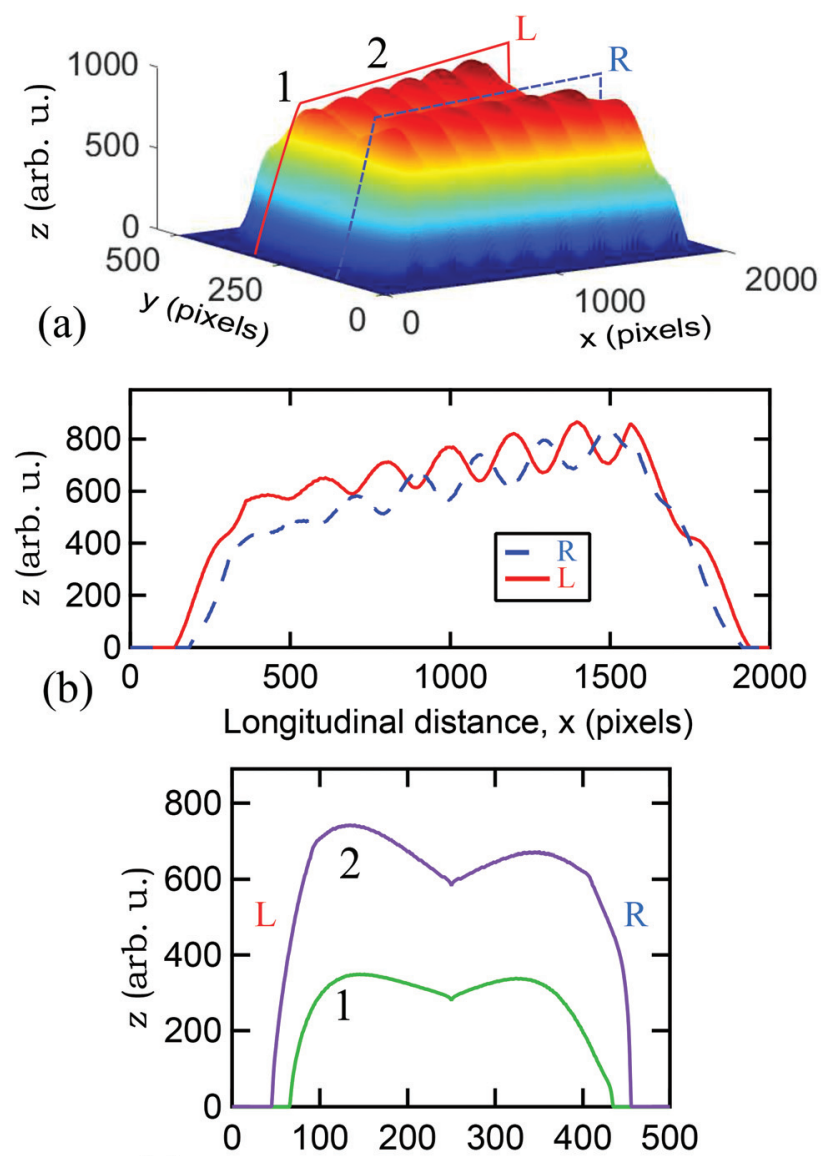

(c) Lateral distance, y (pixels)

Fig. 5 (Color online) (a) Surface plot of simulated nanotrack morphology employing the model described in text. The two axial lines along the simulated nanotrack (one solid red and the other broken blue) indicate the paths of the line height scan data presented in (b), and (c) shows two cross sectional height scans obtained from the locations indicated in (a). The horizontal axes here are given in pixels. these data, namely, the presence of height dip at the center of the nanotrack, forming an M-shape through its cross section. In Fig. 5(c), this feature strongly resembles the AFM data in Fig. 2(d), and likewise becomes more prominent with increasing nanotrack height.

Similar too is the asymmetry of the simulated nanotrack cross section, with very different relative gradients for the left and right sidewalls. For convenience, we allow the steeper wall here to correspond also to the $\mathrm{L}$ side, and it follows that the $\mathrm{R}$ side inherits a flatter top and more rounded edge. An asymmetrical cross section essentially arises because the sweep direction of the rotating beam-like flux, $\alpha$, is not equivalent for opposite sides, relative to lateral motion. For a VLS driven droplet moving with sufficient unidirectional speed, such growth asymmetry becomes inevitable in the presence of a rotating vapor source.

With the steepness of the $\mathrm{R}$ edge reduced, it is expected that the VLS growth dynamics of the R side will be significantly altered (compared to the L side) through elevated sidewall wetting and droplet destabilization. This may account for the heightened disorder observed experimentally for the R side of the nanotrack top (see Fig. 2(b) and (c)).

Within the framework of our model the origins of particular morphological features become evident. The central nanotrack height minimum - creating an M-shape in the cross sectional data - manifests as a consequence of fewer diffusing supply species arriving at the center rear of the moving droplet. As $h$ increases, the frequency of adatoms successfully arriving at the droplet rear from the epitaxial surface is greatly reduced, and the shape becomes more pronounced. Likewise, the presence of strong shadowing effects for the 3D droplet and a rotating beam-like vapor source induces a periodic and out-of-phase height variation along opposite sides of the planar growth. These two aspects of growth are mutually exclusive; without periodic shadowing effects, supply diffusion would only define the M-shape cross section. This offers independently tunable degrees of freedom in the design of planar nanotracks possessing these features.

\section{Conclusion}

We have reported the characterization of self-aligned GaAsBi planar nanotracks exhibiting periodic height variations along their length. Based on a detailed morphological study of the nanotracks, we developed a semi-empirical geometric model to account for three interesting characteristics observed: (i) a cross section resembling an "M-shape", (ii) asymmetrically rising left and right edges, and (iii) a periodic and out-ofphase undulation pattern across the nanotrack growth axis. These features are found to originate from strong diffusion and shadowing effects. The asymmetry in the nanotrack cross section is expected to promote a disorderly growth process for the less sheer sidewall, a feature observed in experiment. It is shown that the assumption of a skewed distribution of enhanced growth rate near the TPB reproduces the nanotrack 
pattern with high precision, implying this approach to be accurate. While diffusion and shadowing effects are typically avoided during MBE growth, we conclude they might actually be utilized in the design and growth of planar nanostructures with controlled periodic non-monotonous structure.

\section{Acknowledgements}

The authors acknowledge support given by the Australian Research Council (ARC) Discovery Project (no. DP140101501) and the research used equipment funded by the ARC Linkage, Infrastructure, Equipment and Facilities (LIEF) grant (no. LE0882813), located at the UOW Electron Microscopy Centre. M. Henini acknowledges King Abdul-Aziz City for Sciences and Technology (KACST) for financial support. The work at University of Arkansas was supported by NSF Career Award no. DMR-1149605.

\section{References}

1 P. Yang, R. Yan and M. Fardy, Nano Lett., 2010, 10, 15291536.

2 R. S. Wagner and W. C. Ellis, Appl. Phys. Lett., 1964, 4, 89-90.

3 N. P. Dasgupta, J. Sun, C. Liu, S. Brittman, S. C. Andrews, J. Lim, H. Gao, R. Yan and P. Yang, Adv. Mater., 2014, 26, 2137-2184.

4 J. M. Redwing, X. Miao and X. Li, Handbook of Crystal Growth, Elsevier, Boston, 2014, pp. 399-439.

5 M. Yoshimoto and K. Oe, Molecular Beam Epitaxy, Elsevier, Oxford, 2013, pp. 159-170.

6 K. Oe, Jpn. J. Appl. Phys., 2002, 41, 2801.

7 M. Ferhat and A. Zaoui, Phys. Rev. B: Condens. Matter, 2006, 73, 115107.

8 T. Fuyuki, R. Yoshioka, K. Yoshida and M. Yoshimoto, Appl. Phys. Lett., 2013, 103, 202105.

9 N. Hossain, I. P. Marko, S. R. Jin, K. Hild, S. J. Sweeney, R. B. Lewis, D. A. Beaton and T. Tiedje, Appl. Phys. Lett., 2012, 100, 051105.

10 G. Vardar, S. W. Paleg, M. V. Warren, M. Kang, S. Jeon and R. S. Goldman, Appl. Phys. Lett., 2013, 102, 042106.

11 M. Dejarld, D. Nothern and J. M. Millunchick, J. Appl. Phys., 2014, 115, 114307.

12 H. Wang, L. A. Zepeda-Ruiz, G. H. Gilmer and M. Upmanyu, Nat. Commun., 2013, 4, 1956.

13 C.-Y. Wen, J. Tersoff, K. Hillerich, M. C. Reuter, J. H. Park, S. Kodambaka, E. A. Stach and F. M. Ross, Phys. Rev. Lett., 2011, 107, 025503.

14 K. W. Schwarz and J. Tersoff, Nano Lett., 2012, 12, 13291332.

15 H. Wang, J.-T. Wang, Z.-X. Cao, W.-J. Zhang, C.-S. Lee, S.-T. Lee and X.-H. Zhang, Nat. Commun., 2014, 6, 6412.

16 D. S. Oliveira, L. H. Tizei, D. Ugarte and M. A. Cotta, Nano Lett., 2012, 13, 9-13.
17 B. Tian, P. Xie, T. J. Kempa, D. C. Bell and C. M. Leiber, Nat. Nanotechnol., 2009, 4, 824-829.

18 K. W. Schwarz, J. Tersoff, S. Kodambaka and F. M. Ross, Phys. Rev. Lett., 2014, 113, 055501.

19 K. W. Schwarz and J. Tersoff, Nano Lett., 2011, 12, 13291332.

20 R. S. Dowdy, C. Zhang, P. K. Mohseni, S. A. Fortuna, J.-G. Wen, J. J. Coleman and X. Li, Opt. Mater. Express, 2013, 3, 1687-1697.

21 D. D. D. Ma, C. S. Lee, F. C. K. Au, S. Y. Tong and S. T. Lee, Science, 2003, 299, 1874-1877.

22 P. B. Sorokin, P. V. Avramov, L. A. Chernozatonskii, D. G. Fedorov and S. G. Ovchinnikov, J. Phys. Chem. A, 2008, 112, 9955-9964.

23 P. J. Kowalczyk, O. Mahapatra, S. A. Brown, G. Bian, X. Wang and T.-C. Chiang, Nano Lett., 2013, 13, 43-47.

24 G. Brönstrup, N. Jahr, C. Leiterer, A. Csáki, W. Fritzsche and S. Christiansen, ACS Nano, 2010, 4, 7113-7122.

25 Y. Zi, K. Jung, D. Zakharov and C. Yang, Nano Lett., 2013, 13, 2786-2791.

26 Y. Y. Cao and G. W. Yang, J. Phys. Chem. C, 2012, 116, 6233-6238.

27 S. Breuer, M. Hilse, A. Trampert, L. Geelhaar and H. Reichert, Phys. Rev. B: Condens. Matter Mater. Phys., 2010, 82, 075406.

28 G. Zhang, K. Tateno, H. Gotoh and H. Nakano, Nanotechnology, 2010, 21, 095607.

29 W. Sun, Y. Guo, H. Xu, Q. Gao, H. H. Tan, C. Jagadish and J. Zou, Appl. Phys. Lett., 2013, 103, 223104.

30 C. Zhang, X. Miao, P. K. Mohseni, W. Choi and X. Li, Nano Lett., 2014, 14, 6836-6841.

31 S. A. Fortuna, J. Wen, I. S. Chun and X. Li, Nano Lett., 2008, 8, 4421-4427.

32 X. Lu, D. A. Beaton, R. B. Lewis, T. Tiedje and Y. Zhang, Appl. Phys. Lett., 2009, 95, 041903.

33 M. Yoshimoto, S. Murata, A. Chayahara, Y. Horino, J. Saraie and K. Oe, Jpn. J. Appl. Phys., 2003, 42, 1235.

34 B. A. Wacaser, K. A. Dick, J. Johansson, M. T. Borgström, K. Deppert and L. Samuelson, Adv. Mater., 2009, 21, 153165.

35 W. Braun, V. M. Kaganer, A. Trampert, H.-P. Schönherr, Q. Gong, R. Nötzel, L. Däweritz and K. H. Ploog, J. Cryst. Growth, 2001, 227, 51-55.

36 R. A. Dowdy, D. A. Walko and X. Li, Nanotechnology, 2013, 24, 035304.

37 J. Wu, Z. M. Wang, A. Z. Li and G. J. Salamo, Phys. Status Solidi RRL, 2010, 4, 371-373.

38 I. Horcas, R. Fernández, J. M. Gómez-Rodríguez, J. Colchero, J. Gómez-Herrero and A. M. Baro, Rev. Sci. Instrum., 2007, 78, 013705.

39 L. Cao, B. Garipcan, J. S. Atchison, C. Ni, B. Nabet and J. E. Spanier, Nano Lett., 2006, 6, 1852.

40 D. Kashchiev, Cryst. Growth Des., 2006, 6, 1154-1156.

41 J. Johansson, C. P. T. Svensson, T. Mårtensson, L. Samuelson and W. Seifert, J. Phys. Chem. B, 2005, 109, 13567-13571. 
42 F. Glas, Phys. Status Solidi B, 2010, 247, 254-258.

43 D. Kashchiev, Nucleation: Basic Theory With Applications, Butterworth-Heinemann, Oxford, 2000.

44 I. V. Markov, Crystal Growth for Beginners: Fundamentals of Nucleation, Crystal Growth and Epitaxy, Hackensack, New Jersey, 2003.

45 B. J. Kim, J. Tersoff, S. Kodambaka, M. C. Reuter, E. A. Stach and F. M. Ross, Science, 2008, 322, 1070-1073.
46 R. P. Sear, J. Phys.: Condens. Matter, 2007, 19, 466106.

47 S. Hofmann, R. Sharma, C. T. Wirth, F. Cervantes-Sodi, C. Ducati, T. Kasama, R. E. Dunin-Borkowski, J. Drucker, P. Bennett and J. Robertson, Nat. Mater., 2008, 7, 372375.

48 J. Tersoff, D. E. Jesson and W. X. Tang, Science, 2009, 324, 236-238. 